Research Article

\title{
Pricing Vulnerable Options in a Mixed Fractional Brownian Motion with Jumps
}

\author{
Panhong Cheng $\mathbb{D}^{1,2}$ and Zhihong $\mathrm{Xu} \mathbb{D}^{3}$ \\ ${ }^{1}$ School of Management, University of Shanghai for Science and Technology, Shanghai 200093, China \\ ${ }^{2}$ School of Mathematics and Finance, Chuzhou University, Chuzhou 239000, China \\ ${ }^{3}$ Public Teaching Department, Rizhao Polytechnic, Rizhao 276826, China \\ Correspondence should be addressed to Panhong Cheng; hollycheng@sina.cn
}

Received 7 June 2021; Revised 23 November 2021; Accepted 10 December 2021; Published 31 December 2021

Academic Editor: Florentino Borondo

Copyright (c) 2021 Panhong Cheng and Zhihong Xu. This is an open access article distributed under the Creative Commons Attribution License, which permits unrestricted use, distribution, and reproduction in any medium, provided the original work is properly cited.

\begin{abstract}
A new framework for pricing European vulnerable options is developed in the case where the underlying stock price and firm value follow the mixed fractional Brownian motion with jumps, respectively. This research uses the actuarial approach to study the pricing problem of European vulnerable options. An analytic closed-form pricing formula for vulnerable options with jumps is obtained. For the purpose of understanding the pricing model, some properties of this pricing model are discussed in the paper. Finally, we compare and analyze the pricing results of different pricing models and discuss the influences of basic parameters on the pricing results of our proposed model by using numerical simulations, and the corresponding economic analyses about these influences are given.
\end{abstract}

\section{Introduction}

Options are popular financial derivatives that play essential roles in financial markets. An option is an agreement that the holder has the right to buy or sell a certain amount of underlying assets at an exercise price during a fixed period. In 1973, a famous option pricing model was introduced by Black and Scholes [1], which paved the way for the wide application of options in financial markets. With the sustainable development of financial markets, there are more and more over-the-counter (in short, OTC) options, and the transaction scales of OTC options are also growing. In the OTC market, both sides of the transaction negotiate in private and make one-to-one transaction, and this market does not guarantee that the counterparty can realize the promise of payments to the option holder, so credit risk, also known as default risk, exists in OTC options trading. When the option holder is subject to credit risk, then the option with default is defined as a vulnerable option. Based on two kinds of credit risk models, structural model and reducedform model, there is enormous literature studying the pricing problem of vulnerable options. Johnson and Stulz [2] first proposed the pricing model of vulnerable options. Since then, the study of vulnerable options has received more attention. For instance, Jarrow and Turnbull [3] studied the pricing of vulnerable options which had the assumption of independence between the options' underlying assets and the default risk of the counterparty by adopting the reducedform model. Klein [4] obtained an analytic pricing formula of vulnerable options by relaxing the independence assumption of the pricing model in Johnson and Stulz [2] and Jarrow and Turnbull [3] and applying the structural model. Hung and Liu [5] extended the model of Klein [4] to price vulnerable options when the market is incomplete.

Motivated by the aforementioned works, some researchers further consider the construction of pricing model on vulnerable options under the conditions of stochastic interest rate, stochastic volatility, and jump risks. For instance, Liao and Huang [6] extended the framework of Klein and Inglis [7] to obtain closed-form pricing formulae for vulnerable options under stochastic interest rate by the riskneutral pricing approach. Yoon and Kim [8] investigated the 
pricing problem of vulnerable options under the Hull-White interest rate and derived an analytic pricing formula for European vulnerable options by using double Mellin transforms. Lee et al. [9] incorporated Heston stochastic volatility model to describe the dynamics of underlying asset and the firm value of the counterparty and provided a closed-form analytic formula of vulnerable options price by using the Green function and Fourier transforms. Wang et al. [10] improved the model in Lee et al. [9]. Taking the differences between the volatility of the underlying asset and that of the asset of the counterparty into consideration, the authors in [10] used two-factor stochastic volatility diffusion processes to capture the changing characteristics of the underlying asset and that of the asset of the counterparty and derived a pricing formula for vulnerable options by applying equivalent martingale measure transformation method. All studies are devoted to constructing a pricing model for vulnerable options by diffusion processes in the above papers. However, owing to jump risk caused by rare events which occurs in the underlying asset and the counterparty asset, some literature proposed jump-diffusion models to consider vulnerable options pricing. Under the jump-diffusion assumptions about the underlying asset and the counterparty asset, $\mathrm{Xu}$ et al. [11] presented an improved method of vulnerable options pricing. Wang [12] carried out research into the pricing problem of vulnerable options by assuming that the underlying asset price and the value of counterparty asset both followed jump-diffusion processes and the default barrier was stochastic. Han et al. [13] investigated vulnerable options pricing considering the market prices of common systematic jump risks under regimeswitching jump-diffusion models and derived explicit analytic pricing formulae for vulnerable options by risk-neutral pricing theory. Under the reduced-form framework, Niu et al. [14] incorporated jump risks and dynamical correlation between the underlying asset and the counterparty asset in vulnerable options to present jump-diffusion pricing models with stochastic correction. More studies about vulnerable options pricing could be seen in Ma et al. [15], Lee and Kim [16], Ma et al. [17], Han [18], Niu and Wang [19], Yang et al. [20], Pasricha and Goel [21], and the references therein.

All the discussions mentioned above demonstrated that the logarithmic returns of the underlying asset were independent and identically distributed normal random variables, as well as the logarithmic returns of the counterparty asset. However, a series of financial empirical studies showed that the distribution of return on financial assets has the features of high peaks, heavy tails, and long-range dependence (see [22-26]). For example, Mandelbrot and Van Ness [22] first found that stock returns have long-range dependence and gave the definition of fractional Brownian motion (in short, FBM). Since then, FBM that exhibits self-similarity and long memory becomes a useful tool for capturing long memory behavior of the financial asset. However, the fractional Brownian motion is neither a Markov process nor a semimartingale (except $H=1 / 2$ ), so the usual stochastic calculus cannot be used to analyze it. Although Lin [27] developed a pathwise integral theory for FBM, Rogers [28] proved that the corresponding market has arbitrage. Duncan et al. [29] defined a new kind of integral based on Wick product that is called fractional Itô integral and derived the fractional Itô integral rule corresponding to the FBM. Hu and Øksendal [30] further studied the integral theory of FBM based on Wick product and showed that the corresponding Itô type fractional Black-Scholes market has no arbitrage under the fractional Itô integral rule, contrary to the situation when the pathwise integral was used in [28]. Moreover, $\mathrm{Hu}$ and Øksendal [30] proved that their Itô fractional Black-Scholes market is complete and derived the pricing formula for a European option at $t=0$. Necula [31] studied the pricing of European options under fractional Brownian motion environment by utilizing fractal geometry theory. Elliott and van der Hoek [32] analyzed the properties of fractional Brownian motion and its application in finance. Nualart [33] proposed a fractional Black-Scholes options pricing model as an improvement of the classical Black-Scholes model. Xiao et al. [34] constructed a new pricing model for European currency option in a fractional Brownian motion with jumps and gave the estimation method of parameters in the pricing model. Under the assumptions of the stock price obeying the fractional jumpdiffusion model and the interest rate and default intensity obeying fractional Vasicek model, Wang et al. [35] derived the pricing formulae for European vulnerable options using the equivalent martingale measure method. Although some research has constructed no arbitrage fractional Black-Scholes model under Wick self-financing strategy, Björk and Hult [36] showed that the fractional market based on Wick product has no arbitrage but the definition of the corresponding self-financing trading strategy is too restrictive. Furthermore, the valuation of options may be negative prices when applying FBM. Thus, FBM based on Wick products is with limited applicability in finance. In order to overcome those problems and take the long-range dependence into account, it is reasonable to use the mixed fractional Brownian motion (in short, MFBM) to characterize the stochastic fluctuations of financial asset price $[37,38]$. The mixed fractional Brownian motion, known as a generalization of FBM, is a family of centered Gaussian processes that is a linear combination of Brownian motion and independent fractional Brownian motion. Cheridito [39] first applied the MFBM to economics. It was proved that MFBM is equivalent to Brownian motion when $H \in(3 / 4,1)$, and hence the mixed fractional market has no arbitrage in [39]. Xiao et al. [40] studied pricing equity warrants in the MFBM environment and illustrated the validity of their proposed algorithm through numerical examples. Shokrollahi and Klllçman [41] developed a new framework for pricing European currency option, where the spot exchange rate obeyed a MFBM with jumps, and made numerical simulations to illustrate the flexibility of their model. Zhang et al. [42] employed stochastic analysis and fuzzy set theory to propose a fuzzy mixed fractional Brownian motion model with jumps. The closed-form solutions for the prices of European options under the fuzzy MFBM environment were derived through arbitrage-free pricing theory. Moreover, the empirical studies were made to illustrate the reasonableness of the proposed pricing model. Considering the long-range 
dependence of the underlying asset returns, $\mathrm{Li}$ and Wang [43] studied the valuation of the bid and ask prices of European options under the MFBM environment and obtained the explicit formulae for the bid and ask prices by using WANG-transform as a distortion function. Zhang et al. [44] assumed that the price of the underlying stock followed a MFBM, got the analytic pricing formulae for the fixed and floating strike geometric Asian power options through a partial differential equation approach, and presented the lower and upper bounds of the prices of fixed and floating strike geometric Asian power options by utilizing interval numbers. In addition, more studies into the pricing problems of options could be seen in [45-47] and the references therein.

The analytic pricing formula of options has been derived usually by utilizing the risk-neutral pricing theory and equivalent martingale measure transformation method as most of the research quoted above has done. Nevertheless, an actuarial approach, turning the pricing problem of vulnerable options into a fair premium determination, might be a better choice which was put forward by Bladt and Rydberg [48] since it would not require the complexity of calculation of the probabilistic techniques. Yan et al. [49] obtained the pricing formulae of European options in the case where the underlying asset price followed Ornstein-Uhlenbeck process by actuarial approach. Shokrollahi and Klllçman [46] investigated the strategy of fair insurance premium actuarial approach for pricing foreign currency option in the MFBM environment with jumps.

Further, to capture jumps in the underlying asset price and the value of counterparty asset and to take the longrange dependence of those financial assets, in this paper, we use the jump process, that is, Poisson process (see Xiao et al. [34]), to characterize the sudden changes and the diffusion process, that is, MFBM, to characterize the normal continuous fluctuations in financial assets. Closed-form formulae for vulnerable options are derived in MFBM environment with jumps by actuarial approach where we adopt the typical structural approach to describe default risk. Then, we illustrate some properties of our proposed pricing model. The comparative results of our model and other available models indicate that our model is closer to the actual market. In addition, vulnerable options values against different parameters changes in our model are investigated.

The remainder of this article is structured as follows. In Section 2, a pricing model for vulnerable options in the mixed fractional Brownian with jumps is constructed, and closed-form formulae are deduced by actuarial approach. Section 3 presents some properties of the pricing formulae. Section 4 gives numerical simulations according to our proposed pricing model. The concluding remarks are drawn in Section 5.

\section{Pricing Model for Vulnerable Options in a Mixed Jump Fractional Brownian Environment}

Considering the long-range dependence and abnormal fluctuations of financial assets, a mixed jump fractional Brownian motion pricing model for vulnerable options combining the MFBM and jump process is constructed. Closed-form pricing formulae are obtained by actuarial approach.

2.1. Basic Setting of the Pricing Model. Consider a complete probability space $\left.\left(\Omega, \mathscr{F}_{,}, \mathscr{F}_{t}\right\}_{t \geq 0}, P\right)$, in which all the random variables and processes below are defined, and the information filtration $\left\{\mathscr{F}_{t}\right\}_{t \geq 0}$ satisfies the usual conditions, such as being monotonic increasing and right continuous. A mixed fractional Brownian motion $M_{t}^{H}$ is a linear combination of Brownian motion $B_{t}$ and fractional Brownian motion $B_{t}^{H}$ with Hurst parameter $H \in(0,1)$, namely,

$$
M_{t}^{H}=\alpha B_{t}+\beta B_{t}^{H},
$$

where $B_{t}$ and $B_{t}^{H}$ are independent, and $\alpha$ and $\beta$ are two real constants such that $(\alpha, \beta) \neq(0,0)$.

The MFBM is a centered Gaussian process with mean zero and the covariance

$$
\begin{aligned}
\operatorname{Cov}\left(M_{t}^{H}, M_{s}^{H}\right)= & \alpha^{2}(t \wedge s) \\
& +\frac{\beta^{2}}{2}\left(t^{2 H}+s^{2 H}-|t-s|^{2 H}\right), \quad s, t>0 .
\end{aligned}
$$

In this paper, we will only consider $H \in(3 / 4,1)$, in order to get rid of arbitrage in the financial markets. Now, to derive the vulnerable options pricing formulae in a mixed jump fractional market, we shall make the following assumptions:

(i) All securities are perfectly divisible, and there are no transaction costs or taxes.

(ii) Security trading is continuous.

(iii) The short-term interest rate is constant during the lifetime of the derivative securities.

(iv) Dividends are not paid during the lifetime of the underlying asset.

(v) There are no riskless arbitrage opportunities.

(vi) The underlying asset price $S_{t}$ and the firm value of the counterparty $V_{t}$ both follow a MFBM with random jumps under the probability measure $P$, respectively. Thus,

$$
\begin{gathered}
\mathrm{d} S_{t}=S_{t}\left(\mu_{s}(t)-\lambda_{S} \theta_{S}\right) \mathrm{d} t+S_{t}\left(\sigma_{S} \mathrm{~d} B_{S}(t)+\sigma_{S}^{H} \mathrm{~d} B_{S}^{H}(t)\right)+S_{t}\left(J_{S}(t)-1\right) \mathrm{d} N_{S}(t), \\
\mathrm{d} V_{t}=V_{t}\left(\mu_{v}(t)-\lambda_{V} \theta_{V}\right) \mathrm{d} t+V_{t}\left(\sigma_{V} \mathrm{~d} B_{V}(t)+\sigma_{V}^{H} \mathrm{~d} B_{V}^{H}(t)\right)+V_{t}\left(J_{V}(t)-1\right) \mathrm{d} N_{V}(t),
\end{gathered}
$$


where $N_{i}(t)$ denotes a Poisson process with rate $\lambda_{i}$, which is $\mathscr{F}_{t}$-adapted. $J_{i}(t)-1$ is a jump size $\left(J_{i}(0)=1\right)$ with the mean $E\left(J_{i}-1\right)=\theta_{i} . J_{i}(t)$ is jump size percent at time $t$ which is a sequence of lognormal, independent, and identically distributed variables with mean $\ln \left(1+\theta_{i}\right)-\left(\sigma_{J i}^{2} / 2\right)$ and variance $\sigma_{J i}^{2}$. The drift $\mu_{i}(t)$ is supposed to be nonrandom function of time $t$. The volatility $\left(\sigma_{i}, \sigma_{i}^{H}\right)$ is assumed to be a positive constant. $B_{i}(t)$ is a standard Brownian motion, and $B_{i}^{H}(t)$ is a fractional Brownian motion with Hurst parameter $H \in(3 / 4,1) . B_{i}(t)$ and $B_{i}^{H}(t)$ are both $\mathscr{F}_{t}$-adapted. Moreover, it is supposed that the Poisson processes $N_{S}(t), N_{V}(t)$; the jump size percent terms $J_{S}(t), J_{V}(t)$; the standard Brownian motion $B_{i}(t)$; and the fractional Brownian motion $B_{i}^{H}(t)$ are independent. $i \in\{S, V\}$ is satisfied throughout the paper. The covariance of $B_{S}(t)$ and $B_{V}(t)$ is $\rho_{S V} t$, and the covariance of $B_{S}^{H}(t)$ and $B_{V}^{H}(t)$ is $\rho_{S V}^{H} t^{2 H}$.

The payoff of a European vulnerable call option and put option at maturity date $T$ are expressed in this paper and are consistent with Klein [4] as follows, respectively:

$$
\begin{aligned}
& C_{T}^{d}=E\left[\left(S_{T}-K\right)^{+}\left(I_{\left\{V_{T} \geq D^{*}\right\}}+\frac{1-\alpha}{D} V_{T} I_{\left\{V_{T}<D^{*}\right\}}\right)\right], \\
& P_{T}^{d}=E\left[\left(K-S_{T}\right)^{+}\left(I_{\left\{V_{T} \geq D^{*}\right\}}+\frac{1-\alpha}{D} V_{T} I_{\left\{V_{T}<D^{*}\right\}}\right)\right],
\end{aligned}
$$

where $K$ is the strike price of the options. $D^{*}$ is a constant default boundary such that no credit loss occurs if $V_{T}$ is greater than $D^{*}$ and the option holder receives full payment. If $V_{T}$ is less than $D^{*}$, a credit loss occurs. In the event of the credit loss, the option holder only obtains the proportion $(1-\alpha / D) V_{T}$ of the nominal claim by the option writer, where $\alpha$ represents the deadweight costs associated with bankruptcy expressed as a percentage of the firm value of the counterparty and $D$ is the value of total liabilities given by $D^{*}$ and an additional liability.

2.2. Actuarial Approach for Pricing Vulnerable Options. In this section, we deal with the pricing problem for European vulnerable options by actuarial approach, when the underlying stock price and firm value obey the SDEs (3) and (4), respectively. The pricing model can be applied not only to the arbitrage-free, equilibrium, and complete markets, but also to the arbitrage, nonequilibrium, and incomplete markets.

Definition 1. Assume that $\beta_{S}(w)$ and $\beta_{V}(w)(w \in[0, T])$ are the expectation return rates of the stochastics processes $\left\{S_{t}, t \geq 0\right\}$ and $\left\{V_{t}, t \geq 0\right\}$ on $t \in[0, T]$, respectively, which are defined as follows:

$$
\begin{gathered}
\frac{E\left(S_{T}\right)}{S_{0}}=\exp \left(\int_{0}^{T} \beta_{S}(w) \mathrm{d} w\right), \\
\frac{E\left(V_{T}\right)}{V_{0}}=\exp \left(\int_{0}^{T} \beta_{V}(w) \mathrm{d} w\right) .
\end{gathered}
$$

Definition 2. Suppose that $C_{0}^{d}$ and $P_{0}^{d}$ are the values of the European vulnerable call and put option at time $t=0$, respectively, whose stock price is $S_{t}$, firm value of the counterparty is $V_{t}$, strike price is $K$, and maturity date is $T$. Then, the values of the European vulnerable options by actuarial approach are presented as follows:

$$
\begin{aligned}
& C_{0}^{d}=E\left[\left(e^{-\int_{0}^{T} \beta_{S}(w) \mathrm{d} w} S_{T}-e^{-r T} K\right)\left(I_{A_{1}, B_{1}}+\frac{1-\alpha}{e^{-r T} D} e^{-\int_{0}^{T} \beta_{V}(w) \mathrm{d} w} V_{T} I_{A_{1}, B_{2}}\right)\right], \\
& P_{0}^{d}=E\left[\left(e^{-r T} K-e^{-\int_{0}^{T} \beta_{S}(w) \mathrm{d} w} S_{T}\right)\left(I_{A_{2}, B_{1}}+\frac{1-\alpha}{e^{-r T} D} e^{-\int_{0}^{T} \beta_{V}(w) \mathrm{d} w} V_{T} I_{A_{2}, B_{2}}\right)\right],
\end{aligned}
$$

where $A_{1}=e^{-\int_{0}^{T} \beta_{S}(w) \mathrm{d} w} S_{T} \geq e^{-r T} K$ and $A_{2}=e^{-\int_{0}^{T} \beta_{S}(w) \mathrm{d} w}$ $S_{T}<e^{-r T} K$ are the essential conditions for performing the European vulnerable call and put option on the maturity date $T$, respectively. $B_{1}=e^{-\int_{0}^{T} \beta_{V}(w) \mathrm{d} w}$ $V_{T} \geq e^{-r T} D^{*}$ and $B_{2}=e^{-\int_{0}^{T} \beta_{V}(w) \mathrm{d} w} V_{T}<e^{-r T} D^{*}$ indicate whether a credit loss occurs or not. $I_{(\bullet)}$ is an indicator function.

Lemma 1. The expectation return rates $\beta_{S}(w)$ and $\beta_{V}(w)$, defined by (6) and (7), respectively, satisfy the following equalities: 


$$
\begin{aligned}
& \int_{0}^{T} \beta_{S}(w) \mathrm{d} w=\int_{0}^{T} \mu_{S}(w) \mathrm{d} w \\
& \int_{0}^{T} \beta_{V}(w) \mathrm{d} w=\int_{0}^{T} \mu_{V}(w) \mathrm{d} w .
\end{aligned}
$$

Proof. By applying mixed fractional Itô formula, it is easy to

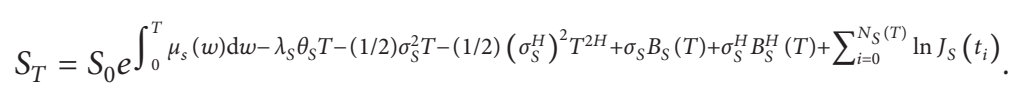

Thus,

$$
\begin{aligned}
\frac{E\left(S_{T}\right)}{S_{0}}= & E\left(e^{\int_{0}^{T} \mu_{s}(w) \mathrm{d} w-\lambda_{S} \theta_{S} T-(1 / 2) \sigma_{S}^{2} T-(1 / 2)\left(\sigma_{S}^{H}\right)^{2} T^{2 H}+\sigma_{S} B_{S}(T)+\sigma_{S}^{H} B_{S}^{H}(T)+\sum_{i=0}^{N_{S}(T)} \ln J_{S}\left(t_{i}\right)}\right) \\
= & e^{\int_{0}^{T} \mu_{S}(w) \mathrm{d} w-\lambda_{S} \theta_{S} T-(1 / 2) \sigma_{S}^{2} T-(1 / 2)\left(\sigma_{S}^{H}\right)^{2} T^{2 H}} \\
& \times E\left[\exp \left(\sigma_{S} B_{S}(T)+\sigma_{S}^{H} B_{S}^{H}(T)\right)\right] E\left[\exp \left(\sum_{i=0}^{N_{S}(T)} \ln J_{S}\left(t_{i}\right)\right)\right] .
\end{aligned}
$$

Since $B_{S}(T) \sim N(0, T), B_{S}^{H}(T) \sim N\left(0, T^{2 H}\right), B_{S}(T)$, and $B_{S}^{H}(T)$ are independent,

$$
E\left[e^{\sigma_{S} B_{S}(T)+\sigma_{S}^{H} B_{S}^{H}(T)}\right]=e^{1 / 2 \sigma_{s}^{2} T+1 / 2\left(\sigma_{S}^{H}\right)^{2} T^{2 H}}
$$

$$
\begin{aligned}
E\left[\exp \left(\sum_{i=0}^{N_{S}(T)} \ln J_{S}\left(t_{i}\right)\right)\right] & =E\left[E\left[\exp \left(\sum_{i=0}^{N_{S}(T)} \ln J_{S}\left(t_{i}\right)\right)\right] \mid N_{S}(T)\right] \\
& =\sum_{m=0}^{\infty} P\left(N_{S}(T)=m\right) E\left[\exp \left(\sum_{i=0}^{m} \ln J_{S}\left(t_{i}\right)\right)\right] \\
& =\sum_{m=0}^{\infty} \frac{\left(\lambda_{S} T\right)^{m}}{m !} e^{-\lambda_{S} T} E\left[\exp \left(\ln J_{S}\left(t_{i}\right)\right)\right]^{m}=\sum_{m=0}^{\infty} \frac{\left(\lambda_{S} T\right)^{m}}{m !} e^{-\lambda_{S} T}\left(1+\theta_{S}\right)^{m}=e^{\lambda_{S} \theta_{S} T}
\end{aligned}
$$

Therefore,

$$
\frac{E\left(S_{T}\right)}{S_{0}}=e^{\int_{0}^{T} \mu_{S}(w) \mathrm{d} w} .
$$

Combining (6) and (16), we have the conclusion (10) of Lemma 1. Similarly, we can prove that equality (11) is satisfied. 
Theorem 1. The prices of European vulnerable options, defined by (8) and (9), are represented as

$$
\begin{aligned}
C_{0}^{d}= & \sum_{m=0}^{\infty} \sum_{n=0}^{\infty} P_{m}\left(\lambda_{S} T\right) P_{n}\left(\lambda_{V} T\right)\left(S_{0} e^{-\lambda_{S} \theta_{S} T}\left(1+\theta_{S}\right)^{m} N_{2}\left(a_{1}(m), a_{2}(n), \rho\right)\right) \\
& -K e^{-r T} N_{2}\left(b_{1}(m), b_{2}(n), \rho\right)+\frac{1-\alpha}{D} S_{0} V_{0} e^{\left(r-\lambda_{S} \theta_{S}-\lambda_{V} \theta_{V}\right) T+\rho \delta_{S} \delta_{V}} \\
& \left(1+\theta_{S}\right)^{m}\left(1+\theta_{V}\right)^{n} N_{2}\left(c_{1}(m),-c_{2}(n),-\rho\right) \\
& \left.-\frac{1-\alpha}{D} K V_{0} e^{-\lambda_{V} \theta_{V} T}\left(1+\theta_{V}\right)^{n} N_{2}\left(d_{1}(m),-d_{2}(n),-\rho\right)\right), \\
P_{0}^{d}= & \sum_{m=0}^{\infty} \sum_{n=0}^{\infty} P_{m}\left(\lambda_{S} T\right) P_{n}\left(\lambda_{V} T\right)\left(K e^{-r T} N_{2}\left(-b_{1}(m), b_{2}(n),-\rho\right)\right. \\
& -S_{0} e^{-\lambda_{S} \theta_{S} T}\left(1+\theta_{S}\right)^{m} N_{2}\left(-a_{1}(m), a_{2}(n),-\rho\right) \\
& +\frac{1-\alpha}{D} K V_{0} e^{-\lambda_{V} \theta_{V} T}\left(1+\theta_{V}\right)^{n} N_{2}\left(-d_{1}(m),-d_{2}(n), \rho\right) \\
& \left.-\frac{1-\alpha}{D} S_{0} V_{0} e^{\left(r-\lambda_{S} \theta_{S}-\lambda_{V} \theta_{V}\right) T+\rho \delta_{S} \delta_{V}} N_{2}\left(-c_{1}(m),-c_{2}(n), \rho\right)\right),
\end{aligned}
$$

where $P_{m}\left(\lambda_{S} T\right)$ and $P_{n}\left(\lambda_{V} T\right)$ are the probability functions of $N_{S}(T)$ and $N_{V}(T)$ throughout the paper, respectively, with $P_{m}\left(\lambda_{S} T\right)=\left(\left(\lambda_{S} T\right)^{m} / m !\right) e^{-\lambda_{S} T}, P_{n}\left(\lambda_{V} T\right)=\left(\left(\lambda_{V} T\right)^{n} / n !\right)$

$$
\begin{aligned}
N_{2}(x, y, \rho) & =\frac{1}{2 \pi \sqrt{1-\rho^{2}}} \int_{-\infty}^{x} \int_{-\infty}^{y} e^{-1 / 2\left(1-\rho^{2}\right)\left(u^{2}-2 \rho u v+v^{2}\right)} \mathrm{d} u \mathrm{~d} v, \\
a_{1}(m) & =b_{1}(m)+\delta_{S}, a_{2}(n)=b_{2}(n)+\rho \delta_{S}, \\
b_{1}(m) & =\frac{\ln \left(S_{0} / K\right)+\left(r-\lambda_{S} \theta_{S}\right) T+m \ln \left(1+\theta_{S}\right)-\left(\delta_{S}^{2} / 2\right)}{\delta_{S}}, b_{2}(n)=\frac{\ln \left(V_{0} / D^{*}\right)+\left(r-\lambda_{V} \theta_{V}\right) T+n \ln \left(1+\theta_{v}\right)-\left(\delta_{V}^{2} / 2\right)}{\delta_{V}}, \\
c_{1}(m) & =b_{1}(m)+\delta_{S}+\rho \delta_{V}, c_{2}(n)=b_{2}(n)+\delta_{V}+\rho \delta_{S}, \\
d_{1}(m) & =b_{1}(m)+\rho \delta_{V}, d_{2}(n)=b_{2}(n)+\delta_{V}, \\
\delta_{S}^{2} & =\sigma_{S}^{2} T+\left(\sigma_{S}^{H}\right)^{2} T^{2 H}+m \sigma_{J S}^{2}, \delta_{V}^{2}=\sigma_{V}^{2} T+\left(\sigma_{V}^{H}\right)^{2} T^{2 H}+n \sigma_{J V}^{2}, \\
\rho & =\frac{\rho_{S V} \sigma_{S} \sigma_{V} T+\rho_{S V}^{H} \sigma_{S}^{H} \sigma_{V}^{H} T^{2 H}}{\delta_{S} \delta_{V}} .
\end{aligned}
$$

$e^{-\lambda_{V} T} \cdot N_{2}$ denotes the bivariate normal cumulative distribution function given by 
where

$$
\begin{aligned}
& E_{1}=E\left(e^{-\int_{0}^{T} \beta_{S}(w) \mathrm{d} w} S_{T} I_{A_{1}, B_{1}}\right), \\
& E_{2}=e^{-r T} K E\left(I_{A_{1}, B_{1}}\right), \\
& E_{3}=\frac{1-\alpha}{e^{-r T} D} E\left(e^{-\int_{0}^{T} \beta_{S}(w) \mathrm{d} w} S_{T} e^{-\int_{0}^{T} \beta_{V}(w) \mathrm{d} w} V_{T} I_{A_{1}, B_{2}}\right), \\
& E_{4}=\frac{(1-\alpha) K}{D} E\left(e^{-\int_{0}^{T} \beta_{V}(w) \mathrm{d} w} V_{T} I_{A_{1}, B_{2}}\right) .
\end{aligned}
$$

Equality (21) illustrates that the value of a European vulnerable call option consists of four terms $E_{1}, E_{2}, E_{3}$, and $E_{4}$. Let

$$
\begin{aligned}
& S_{T}^{m}=S_{0} e^{\int_{0}^{T}} \mu_{S}(w) \mathrm{d} w-\lambda_{S} \theta_{S} T-(1 / 2) \sigma_{S}^{2} T-(1 / 2)\left(\sigma_{S}^{H}\right)^{2} T^{2 H}+\sigma_{S} B_{S}(T)+\sigma_{S}^{H} B_{S}^{H}(T)+\sum_{i=0}^{m} \ln J_{S}\left(t_{i}\right), \\
& V_{T}^{n}=V_{0} e \int_{0}^{T} \mu_{V}(w) \mathrm{d} w-\lambda_{V} \theta_{V} T-(1 / 2) \sigma_{V}^{2} T-(1 / 2)\left(\sigma_{V}^{H}\right)^{2} T^{2 H}+\sigma_{V} B_{V}(T)+\sigma_{V}^{H} B_{V}^{H}(T)+\sum_{i=0}^{n} \ln J_{V}\left(t_{i}\right), \\
& z_{1}=\frac{\sigma_{S} B_{S}(T)+\sigma_{S}^{H} B_{S}^{H}(T)+\sum_{i=0}^{m} \ln J_{S}\left(t_{i}\right)-m \ln \left(1+\theta_{S}\right)+(m / 2) \sigma_{J S}^{2}}{\delta_{S}}, \\
& \delta_{S}^{2}=\sigma_{S}^{2} T+\left(\sigma_{S}^{H}\right)^{2} T^{2 H}+m \sigma_{J S}^{2} \\
& z_{2}=\frac{\sigma_{V} B_{V}(T)+\sigma_{V}^{H} B_{V}^{H}(T)+\sum_{i=0}^{n} \ln J_{V}\left(t_{i}\right)-n \ln \left(1+\theta_{V}\right)+(n / 2) \sigma_{J V}^{2}}{\delta_{V}}, \\
& \delta_{V}^{2}=\sigma_{V}^{2} T+\left(\sigma_{V}^{H}\right)^{2} T^{2 H}+n \sigma_{J V}^{2}, \\
& b_{1}(m)=\frac{\ln \left(S_{0} / K\right)+\left(r-\lambda_{S} \theta_{S}\right) T+m \ln \left(1+\theta_{S}\right)-\left(\delta_{S}^{2} / 2\right)}{\delta_{S}}, \\
& b_{2}(n)=\frac{\ln \left(V_{0} / D^{*}\right)+\left(r-\lambda_{V} \theta_{V}\right) T+n \ln \left(1+\theta_{v}\right)-\left(\delta_{V}^{2} / 2\right)}{\delta_{V}} .
\end{aligned}
$$

Now, we treat each of these terms separately.

Firstly, we calculate the term $E_{2}$.

$$
\begin{aligned}
& E_{2}=e^{-r T} K E\left(I_{A_{1}, B_{1}}\right) \\
& =e^{-r T} K E\left[E\left(I_{A_{1}, B_{1}} \mid N_{S}(T), N_{V}(T)\right)\right] \\
& =e^{-r T} K \sum_{m=0}^{\infty} \sum_{n=0}^{\infty} P_{m}\left(\lambda_{S} T\right) P_{n}\left(\lambda_{V} T\right) \\
& \times E\left(I_{-}^{I} \int_{0}^{T} \beta_{S}(w) \mathrm{d} w_{S_{T}^{m} \geq e^{-r T} K, e}-\int_{0}^{T} \beta_{V}(w) \mathrm{d} w_{V_{T}^{n} \geq e^{-r T} D^{*}}\right) \\
& =e^{-r T} K \sum_{m=0}^{\infty} \sum_{n=0}^{\infty} P_{m}\left(\lambda_{S} T\right) P_{n}\left(\lambda_{V} T\right) E\left(I_{z_{1} \geq-b_{1}(m), z_{2} \geq-b_{2}(n)}\right) \text {, }
\end{aligned}
$$

where equality (23) comes from Lemma 1 and the independence of $B_{i}(T), B_{i}^{H}(T), \quad J_{S}(t), J_{V}(t), N_{S}(T)$, $N_{V}(T), i \in\{S, V\}$.

Note that $\operatorname{cov}\left(B_{S}(T), B_{V}(T)\right)=\rho_{S V} T, \operatorname{cov}\left(B_{S}^{H}(T)\right.$, $\left.B_{V}^{H}(T)\right)=\rho_{S V}^{H} T^{2 H}$; thus,

$$
\left(z_{1}, z_{2}\right) \sim N(0,1 ; 0,1 ; \rho), \rho=\frac{\rho_{S V} \sigma_{S} \sigma_{V} T+\rho_{S V}^{H} \sigma_{S}^{H} \sigma_{V}^{H} T^{2 H}}{\delta_{S} \delta_{V}} \text {. }
$$

Therefore, we obtain

$$
E_{2}=e^{-r T} K \sum_{m=0}^{\infty} \sum_{n=0}^{\infty} P_{m}\left(\lambda_{S} T\right) P_{n}\left(\lambda_{V} T\right) N_{2}\left(b_{1}(m), b_{2}(n), \rho\right) \text {. }
$$

Now, we are in a position to calculate the term $E_{1}$. From Lemma 1 and equality (12), we can get 


$$
\begin{aligned}
& E_{1}=E\left(e^{-\int_{0}^{T} \beta_{S}(w) \mathrm{d} w} S_{T} I_{A_{1}, B_{1}}\right) \\
& =E\left[E\left(e^{-\int_{0}^{T} \beta_{S}(w) \mathrm{d} w} S_{T} I_{A_{1}, B_{1}} \mid N_{S}(T), N_{V}(T)\right)\right] \\
& =\sum_{m=0}^{\infty} \sum_{n=0}^{\infty} P_{m}\left(\lambda_{S} T\right) P_{n}\left(\lambda_{V} T\right)
\end{aligned}
$$

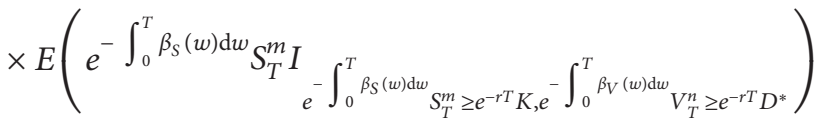

$$
\begin{aligned}
& =S_{0} e^{-\lambda_{S} \theta_{S} T} \sum_{m=0}^{\infty} \sum_{n=0}^{\infty} P_{m}\left(\lambda_{S} T\right) P_{n}\left(\lambda_{V} T\right)\left(1+\theta_{S}\right)^{m} E\left(e^{\delta_{S} z_{1}-\left(\delta_{S}^{2} / 2\right)} I_{z_{1} \geq-b_{1}(m), z_{2} \geq-b_{2}(n)}\right) \\
& =S_{0} e^{-\lambda_{S} \theta_{S} T} \sum_{m=0}^{\infty} \sum_{n=0}^{\infty} P_{m}\left(\lambda_{S} T\right) P_{n}\left(\lambda_{V} T\right)\left(1+\theta_{S}\right)^{m} \int_{-b_{1}(m)}^{+\infty} \int_{-b_{2}(n)}^{+\infty} e^{\delta_{S} z_{1}-\left(\delta_{S}^{2} / 2\right)} \varphi\left(z_{1}, z_{2}, \rho\right) \mathrm{d} z_{1} \mathrm{~d} z_{2} \text {, }
\end{aligned}
$$

where $\varphi\left(z_{1}, z_{2}, \rho\right)$, the standard bivariate normal probability density function, is as follows:

$$
\varphi\left(z_{1}, z_{2}, \rho\right)=\frac{1}{2 \pi \sqrt{1-\rho^{2}}} \mathrm{e}^{-\left(1 / 2\left(1-\rho^{2}\right)\right)\left(z_{1}^{2}+z_{2}^{2}-2 \rho z_{1} z_{2}\right)} .
$$

By using the method of undetermined coefficients, the exponent of the integrand in (26) is of the form

$$
-\frac{1}{2\left(1-\rho^{2}\right)}\left(\left(z_{1}+a\right)^{2}+\left(z_{2}+b\right)^{2}-2 \rho\left(z_{1}+a\right)\left(z_{2}+b\right)\right)+M,
$$

where $\quad a=-\delta_{S}, b=-\rho \delta_{S}, M=0 . \quad$ By setting $a_{1}(m)=b_{1}(m)+\delta_{S}, a_{2}(n)=b_{2}(n)+\rho \delta_{S}, E_{1}$ is given by the formula

$$
\begin{aligned}
E_{1} & =S_{0} \mathrm{e}^{-\lambda_{S} \theta_{S} T} \sum_{m=0}^{\infty} \sum_{n=0}^{\infty} P_{m}\left(\lambda_{S} T\right) P_{n}\left(\lambda_{V} T\right)\left(1+\theta_{S}\right)^{m} \int_{-b_{1}(m)}^{+\infty} \int_{-b_{2}(n)}^{+\infty} \varphi\left(z_{1}-\delta_{S}, z_{2}-\rho \delta_{S}, \rho\right) \mathrm{d} z_{1} \mathrm{~d} z_{2} \\
& =S_{0} \mathrm{e}^{-\lambda_{S} \theta_{S} T} \sum_{m=0}^{\infty} \sum_{n=0}^{\infty} P_{m}\left(\lambda_{S} T\right) P_{n}\left(\lambda_{V} T\right)\left(1+\theta_{S}\right)^{m} N_{2}\left(b_{1}(m)+\delta_{S}, b_{2}(n)+\rho \delta_{S}\right) \\
& =S_{0} \mathrm{e}^{-\lambda_{S} \theta_{S} T} \sum_{m=0}^{\infty} \sum_{n=0}^{\infty} P_{m}\left(\lambda_{S} T\right) P_{n}\left(\lambda_{V} T\right)\left(1+\theta_{S}\right)^{m} N_{2}\left(a_{1}(m), a_{2}(n), \rho\right) .
\end{aligned}
$$

Next, we calculate the term $E_{3}$. Noting that $e^{-\int_{0}^{T} \beta_{V}(w) \mathrm{d} w} V_{T}^{n}<e^{-r T} D^{*}$ is equivalent to $z_{2}<-b_{2}(n)$, we deduce that

$$
\begin{aligned}
E_{3}= & \frac{1-\alpha}{e^{-r T} D} E\left(e^{-\int_{0}^{T} \beta_{S}(w) \mathrm{d} w} S_{T} e^{-\int_{0}^{T} \beta_{V}(w) \mathrm{d} w} V_{T I_{A_{1}, B_{2}}}\right) \\
= & \frac{(1-\alpha) S_{0} V_{0} e^{r T-\left(\lambda_{S} \theta_{S}+\lambda_{V} \theta_{V}\right) T}}{D} \sum_{m=0}^{\infty} \sum_{n=0}^{\infty} P_{m}\left(\lambda_{S} T\right) P_{n}\left(\lambda_{V} T\right)\left(1+\theta_{S}\right)^{m}\left(1+\theta_{V}\right)^{n} \\
& \times \int_{-b_{1}(m)}^{+\infty} \int_{-\infty}^{-b_{2}(n)} e^{\delta_{S} z_{1}+\delta_{V} z_{2}-\left(\delta_{S}^{2}+\delta_{V}^{2} / 2\right)} \varphi\left(z_{1}, z_{2}, \rho\right) \mathrm{d} z_{1} \mathrm{~d} z_{2}
\end{aligned}
$$




$$
\begin{aligned}
= & \frac{(1-\alpha) S_{0} V_{0} e^{r T-\left(\lambda_{S} \theta_{S}+\lambda_{V} \theta_{V}\right) T+\rho \delta_{S} \delta_{V}}}{D} \sum_{m=0}^{\infty} \sum_{n=0}^{\infty} P_{m}\left(\lambda_{S} T\right) P_{n}\left(\lambda_{V} T\right)\left(1+\theta_{S}\right)^{m}\left(1+\theta_{V}\right)^{n} \\
& \times \int_{-b_{1}(m)}^{+\infty} \int_{-\infty}^{-b_{2}(n)} \varphi\left(z_{1}-\delta_{S}-\rho \delta_{V}, z_{2}-\delta_{V}-\rho \delta_{S}, \rho\right) \mathrm{d} z_{1} \mathrm{~d} z_{2} .
\end{aligned}
$$

Setting $c_{1}(m)=b_{1}(m)+\delta_{S}+\rho \delta_{V}$ and $c_{2}(n)=b_{2}(n)$ $+\delta_{V}+\rho \delta_{S}$, we have

$$
\begin{aligned}
E_{3}= & \frac{(1-\alpha) S_{0} V_{0} \mathrm{e}^{r T-\left(\lambda_{S} \theta_{S}+\lambda_{V} \theta_{V}\right) T+\rho \delta_{S} \delta_{V}}}{D} \sum_{m=0}^{\infty} \sum_{n=0}^{\infty} P_{m}\left(\lambda_{S} T\right) P_{n}\left(\lambda_{V} T\right) \\
& \times\left(1+\theta_{S}\right)^{m}\left(1+\theta_{V}\right)^{n} N_{2}\left(c_{1}(m),-c_{2}(n),-\rho\right) .
\end{aligned}
$$

Finally, we deal with the term $E_{4}$. Similar to calculating $E_{1}$, it is easy to obtain

$$
\begin{aligned}
E_{4}= & \frac{(1-\alpha) K}{D} E\left(e^{-\int_{0}^{T} \beta_{V}(w) \mathrm{d} w} V_{T} I_{A_{1}, B_{2}}\right) \\
= & \frac{(1-\alpha) K V_{0} \mathrm{e}^{-\lambda_{V} \theta_{V} T}}{D} \sum_{m=0}^{\infty} \sum_{n=0}^{\infty} P_{m}\left(\lambda_{S} T\right) P_{n}\left(\lambda_{V} T\right)\left(1+\theta_{V}\right)^{n} \\
& \times \int_{-b_{1}(m)}^{+\infty} \int_{-\infty}^{-b_{2}(n)} \varphi\left(z_{1}-\rho \delta_{V}, z_{2}-\delta_{V}, \rho\right) \mathrm{d} z_{1} \mathrm{~d} z_{2} .
\end{aligned}
$$

Setting $d_{1}(m)=b_{1}(m)+\rho \delta_{V}$ and $d_{2}(n)=b_{2}(n)+\delta_{V}$, we have

$$
E_{4}=\frac{(1-\alpha) K V_{0} e^{-\lambda_{V} \theta_{V} T}}{D} \sum_{m=0}^{\infty} \sum_{n=0}^{\infty} P_{m}\left(\lambda_{S} T\right) P_{n}\left(\lambda_{V} T\right)\left(1+\theta_{V}\right)^{n} N_{2}\left(d_{1}(m),-d_{2}(n),-\rho\right)
$$

Combining everything together, (17) is satisfied. The proof of (18) is the same.

\section{Properties of Pricing Formula}

In the previous section, we obtain the pricing formulae of European vulnerable options. In this section, we will discuss the properties of the pricing formulae.
Remark 1. The pricing formulae (17) and (18) are both convergent.

It is clear that $0 \leq N_{2}(x, y, \rho) \leq 1$. Then, in order to illustrate the convergence of (17), we only need to show the convergence of these series

$$
\begin{aligned}
& \sum_{m=0}^{\infty} \sum_{n=0}^{\infty} P_{m}\left(\lambda_{S} T\right) P_{n}\left(\lambda_{V} T\right) S_{0} \mathrm{e}^{-\lambda_{S} \theta_{S} T}\left(1+\theta_{S}\right)^{m} \\
& \sum_{m=0}^{\infty} \sum_{n=0}^{\infty} P_{m}\left(\lambda_{S} T\right) P_{n}\left(\lambda_{V} T\right) \frac{1-\alpha}{D} K V_{0} \mathrm{e}^{-\lambda_{V} \theta_{V} T}\left(1+\theta_{V}\right)^{n} \\
& \sum_{m=0}^{\infty} \sum_{n=0}^{\infty} P_{m}\left(\lambda_{S} T\right) P_{n}\left(\lambda_{V} T\right) K \mathrm{e}^{-r T} \\
& \sum_{m=0}^{\infty} \sum_{n=0}^{\infty} P_{m}\left(\lambda_{S} T\right) P_{n}\left(\lambda_{V} T\right) \frac{1-\alpha}{D} S_{0} V_{0} \mathrm{e}^{\left(r-\lambda_{S} \theta_{S}-\lambda_{V} \theta_{V}\right) T+\rho \sqrt{\delta_{S} \delta_{V}}}\left(1+\theta_{S}\right)^{m}\left(1+\theta_{V}\right)^{n}
\end{aligned}
$$

Here, we show that $\sum_{m=0}^{\infty} \sum_{n=0}^{\infty} P_{m}\left(\lambda_{S} T\right) P_{n}\left(\lambda_{V} T\right)$ $S_{0} \mathrm{e}^{-\lambda_{S} \theta_{S} T}\left(1+\theta_{S}\right)^{m}$ is convergent. 


$$
\begin{aligned}
& \sum_{m=0}^{\infty} \sum_{n=0}^{\infty} P_{m}\left(\lambda_{S} T\right) P_{n}\left(\lambda_{V} T\right) S_{0} e^{-\lambda_{S} \theta_{S} T}\left(1+\theta_{S}\right)^{m} \\
& =\sum_{m=0}^{\infty} P_{m}\left(\lambda_{S} T\right) S_{0} e^{-\lambda_{S} \theta_{S} T}\left(1+\theta_{S}\right)^{m} \sum_{n=0}^{\infty} P_{n}\left(\lambda_{V} T\right) \\
& =\sum_{m=0}^{\infty} \frac{e^{-\lambda_{S}\left(1+\theta_{S}\right) T} S_{0}}{m !}\left(\lambda_{S} T\left(1+\theta_{S}\right)\right)^{m} .
\end{aligned}
$$

Setting $u_{m}=\left(e^{-\lambda_{S}\left(1+\theta_{S}\right) T} S_{0} / m !\right)\left(\lambda_{S} T\left(1+\theta_{S}\right)\right)^{m}$, we have

$$
\lim _{m \longrightarrow \infty}\left|\frac{u_{m+1}}{u_{m}}\right|=\lim _{m \longrightarrow \infty} \frac{\lambda_{S} T\left(1+\theta_{S}\right)}{m+1}=0<1 .
$$

According to D'Alembert's test, $\sum_{m=0}^{\infty} \sum_{n=0}^{\infty} P_{m}\left(\lambda_{S} T\right)$ $P_{n}\left(\lambda_{V} T\right) S_{0} e^{-\lambda_{S} \theta_{S} T}\left(1+\theta_{S}\right)^{m}$ is convergent.

The convergence of the other three series will be proved in the same way. Therefore, the pricing formula (17) is convergent.

Similarly, the convergence of (18) can be shown.

Remark 2. If there is no risk of bankruptcy, i.e., $D^{*} \longrightarrow 0$ and $\sigma_{S}^{H}=0$, the option value is similar to the Merton jumpdiffusion model in [50]. Here, the pricing formulae for European call and put options under Merton jump-diffusion model are as follows:

$$
\begin{aligned}
& C_{0}=\sum_{m=0}^{\infty} P_{m}\left(\lambda_{S} T\right)\left(S_{0} e^{-\lambda_{S} \theta_{S} T}\left(1+\theta_{S}\right)^{m} N\left(\widehat{a_{1}}(m)\right)-K e^{-r T} N\left(\widehat{b_{1}}(m)\right)\right), \\
& P_{0}=\sum_{m=0}^{\infty} P_{m}\left(\lambda_{S} T\right)\left(K e^{-r T} N\left(-\widehat{b_{1}}(m)\right)-S_{0} e^{-\lambda_{S} \theta_{S} T}\left(1+\theta_{S}\right)^{m} N\left(-\widehat{a_{1}}(m)\right)\right),
\end{aligned}
$$

where $\quad \widehat{a}_{1}(m)=\widehat{b}_{1}(m)+{\widehat{\delta_{S}}}_{S_{2}} \quad \widehat{b}_{1}(m)=\left(\ln \left(S_{0} / K\right)+\right.$ $\left.\left(r-\lambda_{S} \theta_{S}\right) T+m \ln \left(1+\theta_{S}\right)-\left(\widehat{\delta}_{S} / 2\right)\right) / \widehat{\delta}_{S}, \widehat{\delta}_{S}^{2}=\sigma_{S}^{2} T+m \sigma_{J S}^{2}$, and $N(\bullet)$ denotes the standard normal cumulative distribution function.

It is easily seen that $a_{2} \longrightarrow+\infty, b_{2} \longrightarrow+\infty, c_{2}$ $\longrightarrow+\infty, d_{2} \longrightarrow+\infty$ as $D^{*} \longrightarrow 0$ in the pricing formulae (17) and (18). Note that $N_{2}(z,-\infty, \rho) \longrightarrow 0, N_{2}$ $(z,+\infty, \rho) \longrightarrow N(z)$; then, (17) and (18) can be simplified to (37) and (38), respectively.

Moreover, if no jump occurs in the stock price, our model will reduce to the Black-Scholes model in [1].
Formulae (17) and (18) can be expressed as the following pricing formulae for European call and put options:

$$
\begin{aligned}
& C_{0}=S_{0} N(a)-K e^{-r T} N(b), \\
& P_{0}=K e^{-r T} N(-b)-S_{0} N(-a),
\end{aligned}
$$

where $a=b+\sigma_{S} \sqrt{T}, b=\left(\ln \left(S_{0} / K\right)+\left(r-\left(\sigma_{S}^{2} / 2\right)\right) T\right) / \sigma_{S} \sqrt{T}$.

Remark 3. The basic diffusion model which was proposed by Klein [4] is a special case of our model in this article when $\sigma_{S}^{H}=\sigma_{V}^{H}=0, \lambda_{s}=\lambda_{v}=0, m=n=0$. Here, the pricing formulae (17) and (18) can be rewritten as follows:

$$
\begin{aligned}
C_{0}^{d}= & S_{0} N_{2}\left(a_{1}, a_{2}, \rho_{S V}\right)-K e^{-r T} N_{2}\left(b_{1}, b_{2}, \rho_{S V}\right) \\
& +\frac{1-\alpha}{D} S_{0} V_{0} e^{r T+\rho_{S V} \sigma_{S} \sigma_{V} T} N_{2}\left(c_{1},-c_{2},-\rho_{S V}\right)-\frac{1-\alpha}{D} K V_{0} N_{2}\left(d_{1},-d_{2},-\rho_{S V}\right), \\
P_{0}^{d}= & K e^{-r T} N_{2}\left(-b_{1}, b_{2},-\rho_{S V}\right)-S_{0} N_{2}\left(-a_{1}, a_{2},-\rho_{S V}\right) \\
& +\frac{1-\alpha}{D} K V_{0} N_{2}\left(-d_{1},-d_{2}, \rho_{S V}\right)-\frac{1-\alpha}{D} S_{0} V_{0} e^{r T+\rho_{S V} \sigma_{S} \sigma_{V} T} N_{2}\left(-c_{1},-c_{2}, \rho_{S V}\right),
\end{aligned}
$$

where 


$$
\begin{aligned}
& a_{1}=b_{1}+\sigma_{S} \sqrt{T}, \\
& a_{2}=b_{2}+\rho_{S V} \sigma_{S} \sqrt{T}, \\
& b_{1}=\frac{\ln \left(S_{0} / K\right)+\left(r-\left(\sigma_{S}^{2} / 2\right)\right) T}{\sigma_{S} \sqrt{T}}, \\
& b_{2}=\frac{\ln \left(V_{0} / D^{*}\right)+\left(r-\left(\sigma_{V}^{2} / 2\right)\right) T}{\sigma_{V} \sqrt{T}}, \\
& c_{1}=b_{1}+\sigma_{S} \sqrt{T}+\rho_{S V} \sigma_{V} \sqrt{T}, \\
& c_{2}=b_{2}+\sigma_{V} \sqrt{T}+\rho_{S V} \sigma_{S} \sqrt{T}, \\
& d_{1}=b_{1}+\rho_{S V} \sigma_{V} \sqrt{T}, \\
& d_{2}=b_{2}+\sigma_{V} \sqrt{T} .
\end{aligned}
$$

Remark 4. When the volatility $\sigma_{S}^{H}=\sigma_{V}^{H}=0, \lambda_{V}=0, n=0$, our jump mixed fractional motion model for pricing vulnerable options will reduce to the jump-diffusion model in the Brownian motion environment which was studied by $\mathrm{Xu}$ et al. [51].

Remark 5. Assume that the stock price pays a continuous dividend yield $q$; then, the pricing formulae of vulnerable options can be given when $S_{0}$ is replaced with $S_{0} e^{-q T}$ in (17) and (18), and $r$ is replaced with $r-q$ in the first set of parameters to the bivariate normal distribution function. (the $a_{k}, b_{k}, c_{k}, d_{k}$ term, $k=1$ ). With those adjustments, the equations are presented as follows:

$$
\begin{aligned}
C_{0}^{d}= & \sum_{m=0}^{\infty} \sum_{n=0}^{\infty} P_{m}\left(\lambda_{S} T\right) P_{n}\left(\lambda_{V} T\right)\left(S_{0} e^{-\left(q+\lambda_{S} \theta_{S}\right) T}\left(1+\theta_{S}\right)^{m} N_{2}\left(\tilde{a_{1}}(m), \tilde{a_{2}}(n), \rho\right)\right. \\
& -K e^{-r T} N_{2}\left(\tilde{b_{1}}(m), \tilde{b_{2}}(n), \rho\right)+\frac{1-\alpha}{D} S_{0} V_{0} e^{\left(r-q-\lambda_{S} \theta_{S}-\lambda_{V} \theta_{V}\right) T+\rho \delta_{S} \delta_{V}} \\
& \left(1+\theta_{S}\right)^{m}\left(1+\theta_{V}\right)^{n} N_{2}\left(\tilde{c_{1}}(m),-\tilde{c_{2}}(n),-\rho\right) \\
& \left.-\frac{1-\alpha}{D} K V_{0} e^{-\lambda_{V} \theta_{V} T}\left(1+\theta_{V}\right)^{n} N_{2}\left(\widetilde{d_{1}}(m),-\tilde{d_{2}}(n),-\rho\right)\right) \\
P_{0}^{d}= & \sum_{m=0}^{\infty} \sum_{n=0}^{\infty} P_{m}\left(\lambda_{S} T\right) P_{n}\left(\lambda_{V} T\right)\left(K e^{-r T} N_{2}\left(-\tilde{b_{1}}(m), \tilde{b_{2}}(n),-\rho\right)\right. \\
& -S_{0} e^{-\left(q+\lambda_{S} \theta_{S}\right) T}\left(1+\theta_{S}\right)^{m} N_{2}\left(-\tilde{a_{1}}(m), \tilde{a_{2}}(n),-\rho\right) \\
& +\frac{1-\alpha}{D} K V_{0} e^{-\lambda_{V} \theta_{V} T}\left(1+\theta_{V}\right)^{n} N_{2}\left(-\tilde{d_{1}}(m),-\tilde{d}_{2}(n), \rho\right) \\
& \left.-\frac{1-\alpha}{D} S_{0} V_{0} e^{\left(r-q-\lambda_{S} \theta_{S}-\lambda_{V} \theta_{V}\right) T+\rho \delta_{S} \delta_{V}}\left(1+\theta_{S}\right)^{m}\left(1+\theta_{V}\right)^{n} N_{2}\left(-\tilde{c_{1}}(m),-\tilde{c_{2}}(n), \rho\right)\right)
\end{aligned}
$$

where

$$
\begin{aligned}
& \tilde{a}_{1}(m)=\tilde{b_{1}}(m)+\delta_{S}, \tilde{a}_{2}(n)=\tilde{b}_{2}(n)+\rho \delta_{S}, \\
& \tilde{b_{1}}(m)=\frac{\ln \left(S_{0} / K\right)+\left(r-q-\lambda_{S} \theta_{S}\right) T+m \ln \left(1+\theta_{S}\right)-\left(\delta_{S}^{2} / 2\right)}{\delta_{S}}, \\
& \tilde{b_{2}}(n)=\frac{\ln \left(V_{0} / D^{*}\right)+\left(r-\lambda_{V} \theta_{V}\right) T+n \ln \left(1+\theta_{v}\right)-\left(\delta_{V}^{2} / 2\right)}{\delta_{V}}, \\
& \tilde{c_{1}}(m)=\tilde{b}_{1}(m)+\delta_{S}+\rho \delta_{V}, \tilde{c_{2}}(n)=\tilde{b}_{2}(n)+\delta_{V}+\rho \delta_{S}
\end{aligned}
$$




$$
\begin{aligned}
\widetilde{d}_{1}(m) & ={\widetilde{b_{1}}}(m)+\rho \delta_{V},{\widetilde{d_{2}}}_{2}(n)={\widetilde{b_{2}}}(n)+\delta_{V} \\
\delta_{S}^{2} & =\sigma_{S}^{2} T+\left(\sigma_{S}^{H}\right)^{2} T^{2 H}+m \sigma_{J S}^{2}, \delta_{V}^{2}=\sigma_{V}^{2} T+\left(\sigma_{V}^{H}\right)^{2} T^{2 H}+n \sigma_{J V}^{2}, \\
\rho & =\frac{\rho_{S V} \sigma_{S} \sigma_{V} T+\rho_{S V}^{H} \sigma_{S}^{H} \sigma_{V}^{H} T^{2 H}}{\delta_{S} \delta_{V}} .
\end{aligned}
$$

The above jump-diffusion model with dividend based on MFBM can be used to value currency options. A pricing model for currency options with jumps could be seen in references [41, 46]. It is a special case of the jump-diffusion model with dividend when $q=r_{f}, \lambda_{v}=0, n=0$, and $D^{*} \longrightarrow 0$. Here, the European call and put currency options at time $t=0$ are as follows, respectively:

$$
\begin{aligned}
& C_{0}=\sum_{m=0}^{\infty} P_{m}\left(\lambda_{S} T\right)\left(S_{0} e^{-\left(r_{f}+\lambda_{S} \theta_{S}\right) T}\left(1+\theta_{S}\right)^{m} N\left(\tilde{a_{1}}(m)\right)-K e^{-r T} N\left(\tilde{b_{1}}(m)\right)\right), \\
& P_{0}=\sum_{m=0}^{\infty} P_{m}\left(\lambda_{S} T\right)\left(K e^{-r T} N\left(-\tilde{b_{1}}(m)\right)-S_{0} e^{-\left(r_{f}+\lambda_{S} \theta_{S}\right) T}\left(1+\theta_{S}\right)^{m} N\left(-\tilde{a_{1}}(m)\right)\right),
\end{aligned}
$$

where $\tilde{a_{1}}(m)$ and $\tilde{b_{1}}(m)$ are given above. Moreover, when no jumps occur in stock price, the above pricing formulae (42) and (43) can be simplified to the following equations which are consistent with the conclusion obtained in [45].

$$
\begin{aligned}
& C_{0}=S_{0} e^{-r_{f} T} N(c)-K e^{-r T} N(d), \\
& P_{0}=K e^{-r T} N(-d)-S_{0} e^{-r_{f} T} N(-c),
\end{aligned}
$$

where

$$
\begin{aligned}
& c=d+\sqrt{\sigma_{S}^{2} T+\left(\sigma_{S}^{H}\right)^{2} T^{2 H}}, \\
& d=\frac{\ln \left(S_{0} / K\right)+\left(r-r_{f}\right) T-(1 / 2)\left(\sigma_{S}^{2} T+\left(\sigma_{S}^{H}\right)^{2} T^{2 H}\right)}{\sqrt{\sigma_{S}^{2} T+\left(\sigma_{S}^{H}\right)^{2} T^{2 H}}} .
\end{aligned}
$$

\section{Numerical Analysis}

This section aims to present numerical results of European vulnerable options with jumps given by (17) and (18). For these purposes, we carry out two sets of numerical experiments. The first set is the comparisons with theoretical prices under different models: Klein's model (1996), Black-Scholes' model (1973), and our mixed jump fractional Brownian motion model (hereafter MJFBM). The purpose of these three models we chose is to investigate the effect of jump risk on the values of vulnerable options. The second set is used to analyze the prices of vulnerable options for different parameters under MJFBM. To implement our analysis, the set of basic parameters are given in Table 1, unless otherwise stated. Specifically, the parameters $S_{0}, K, V_{0}, \sigma_{S}, \sigma_{V}, \rho_{S V}, r, D, D^{*}, T$, and $\alpha$ are based on the values reported by Klein [4].

4.1. Comparisons with Option Prices. Pricing results for call options under different pricing models are presented in
Table 2, where $P_{J-M F}$ denotes the prices calculated according to the MJFBM, $P_{K}$ denotes the prices computed by Klein's model, $P_{B S}$ denotes the prices computed by Black-Scholes' model. Since negative jumps in underlying asset reduce the stock prices, and negative jumps in firm values increase the likelihood of default, the call option holder will face an unfavorable situation. From Table 2, we can see that the option prices of the proposed model are smaller than those of Klein's model. This numerical analysis shows that a jump process has a significant impact on option pricing. Using jump-diffusion processes to characterize the fluctuations of the underlying asset prices and firm values is necessary, and the introduction of jump process can make the vulnerable option pricing more accurate. Moreover, due to the effect of default risk, the prices of the proposed model and Klein's model are both lower than the prices of the classical Black-Scholes' model.

4.2. Influence of Parameters. In order to further understand the performance of our pricing model, we carry out the sensitivity analysis in order to investigate the price changes of European vulnerable options with respect to different parameters including initial value of firm, default barrier, ratio of bankruptcy costs, and Hurst index in our model.

Figure 1(a) illustrates how the vulnerable call option prices change with the initial firm value of the counterparty. We can see that the option prices increase with the rising of the initial firm value $V_{0}$. Figure 1(b) shows the option prices against the default boundary $D^{*}$. We observe that option prices are a decreasing function of $D^{*}$. The reason for this observation is that as the default boundary $D^{*}$ increases, the likelihood of default increases and then the option prices decrease. Figure 1(c) describes the relationship between the option prices and the ratio of bankruptcy $\operatorname{costs} \alpha$. We notice that the option prices obtained by the proposed model 
TABLE 1: Parameter values of European vulnerable call option in the base case.

\begin{tabular}{lccc}
\hline Parameter & Value & Parameter & Value \\
\hline Initial value of $S$ & $S_{0}=40$ & Initial value of $V$ & $V_{0}=5$ \\
Strike price & $K=40$ & Interest rate & $r=0.4833$ \\
Value of total liabilities & $D=5$ & Default boundary & $D^{*}=5$ \\
Ratio of bankruptcy costs & $\alpha=0.5$ & Time to maturity & $T=0.3333$ \\
Volatility of $S$ & $\sigma_{S}=0.3$ & Volatility of $V$ & $\sigma_{V}=0.3$ \\
Volatility of $S$ under FBM & $\sigma_{S}^{H}=0.3$ & $\sigma_{V}^{H}=0.3$ \\
Correlation coefficient between $B_{S}(t)$ and $B_{V}(t)$ & $\rho_{S V}=0.5$ & Correlation coefficient between $B_{S}^{H}(t)$ and $B_{V}^{H}(t)$ & $\rho_{S V}^{H}=0.5$ \\
Jump intensity of $S$ & $\lambda_{S}=5$ & Jump intensity of $V$ & $\lambda_{V}=5$ \\
Mean jump size of $S$ & $\theta_{S}=-0.3$ & Mean jump size of $V$ & $\theta_{V}=-0.3$ \\
Standard deviation of $\ln J_{S}(t)$ & $\sigma_{J S}=0.15$ & Standard deviation of $\ln J_{V}(t)$ & $\sigma_{J V}=0.15$ \\
Jump frequency of $S$ & $m=1$ & Jump frequency of $V$ & $n=1$ \\
\hline
\end{tabular}

TABLE 2: European vulnerable call option pricing under different models.

\begin{tabular}{lccc}
\hline Underlying asset prices & \multicolumn{2}{c}{ Different pricing results } \\
& $P_{J-M F}$ & $P_{K}$ & 0.7487 \\
30 & 0.3692 & 0.7372 & 1.3989 \\
32 & 0.5053 & 1.3700 & 2.3237 \\
34 & 1.6597 & 2.2616 & 3.5163 \\
36 & 2.8285 & 3.3935 & 4.9432 \\
38 & 4.0102 & 4.7546 & 6.5571 \\
40 & 5.2011 & 6.2464 & 8.3089 \\
42 & 6.3959 & 7.8606 & 10.1560 \\
44 & 8.5957 & 9.5403 & 12.0653 \\
46 & 10.8006 & 11.2477 & 14.0132 \\
48 & 11.0067 & 12.9415 & 15.9842 \\
50 & 12.2138 & 14.5758 & \\
\hline
\end{tabular}

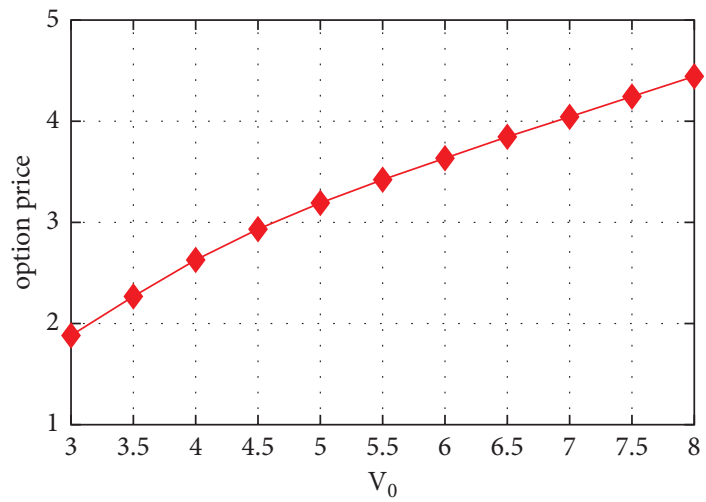

(a)

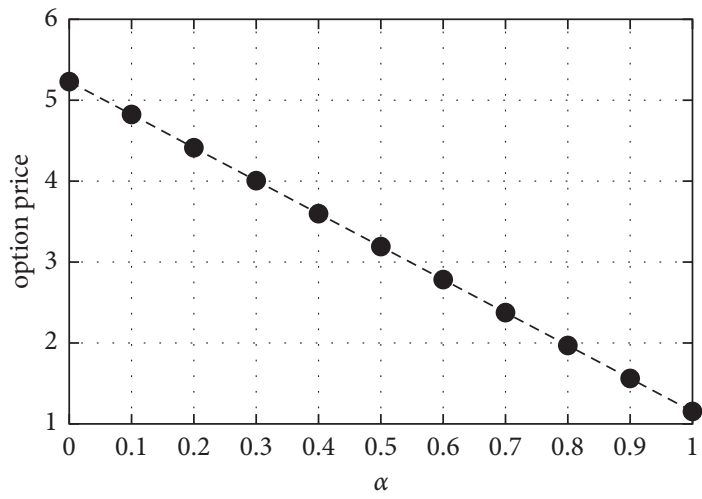

(c)

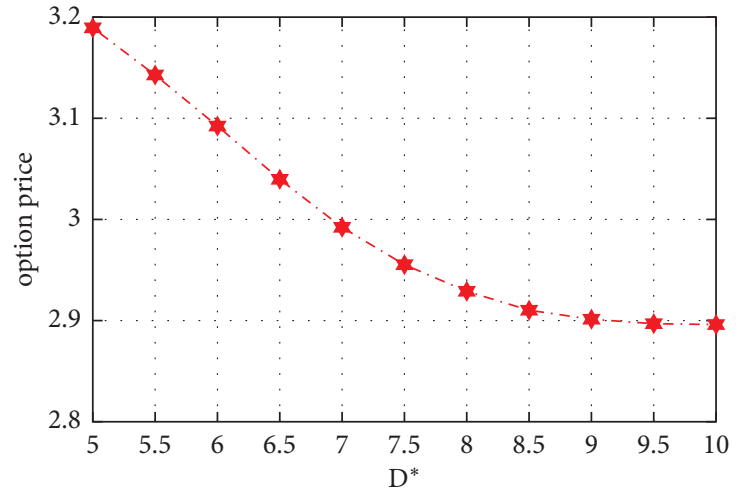

(b)

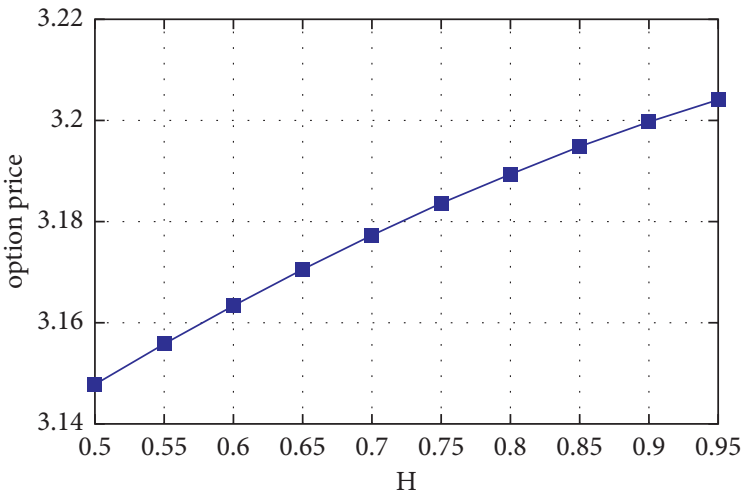

(d)

FIGURE 1: Option price against different parameters: (a) option price against the initial value of $V$; (b) option price against default boundary $D^{*}$; (c) option price against the ratio of bankruptcy cost $\alpha$; (d) option price against Hurst index $H$. 
decrease sharply for the high ratio of bankruptcy costs $\alpha$. This agrees with the financial truth that as the ratio of bankruptcy costs $\alpha$ increases, the recovery rate $(1-\alpha / D) V_{T}$ decreases, and then the expected payoff of the option holder at maturity time $T$ decreases; hence, the option prices decrease. From Figure 1(d), we can observe that the option prices have increasing trends as Hurst parameter $H$ increases. This shows that the Hurst parameter has an important impact on option pricing.

\section{Conclusions}

Reasonable option pricing can not only improve the operation efficiency of the financial markets, but also help investors make effective decisions in the complex and changeable financial markets, so as to maximize their own returns. Since a mixed fractional Brownian motion is an important stochastic process to capture long-range dependence memory of financial phenomena, and jump risks caused by rare events occurring in the underlying stock price and firm value cannot be ignored in the financial markets, we use a mixed jump fractional Brownian motion to capture the behavior characteristics of the stock price and firm value. The actuarial approach turns the pricing problem of vulnerable options into a fair premium determination, which does not require the complexity of the calculation as in the probabilistic techniques. On the basis of actuarial approach, a closed-form pricing formula of European vulnerable options in the MJFBM environment is deduced. Furthermore, some properties of the vulnerable options pricing formulae are derived. Moreover, numerical results in Section 4 show that our mixed jump fractional pricing model is an efficient model for pricing vulnerable options and that some parameters in our model have great influences on the pricing results.

\section{Data Availability}

The data used to support the findings of this research are available from the corresponding author upon request.

\section{Conflicts of Interest}

The authors declare that they have no conflicts of interest.

\section{Acknowledgments}

This research was supported by the Natural Science Foundation of Universities of Anhui Province (KJ2018A0429).

\section{References}

[1] F. Black and M. Scholes, "The pricing of options and corporate liabilities," Journal of Political Economy, vol. 81, no. 3, pp. 637-654, 1973.

[2] H. Johnson and R. Stulz, "The pricing of options with default risk," The Journal of Finance, vol. 42, no. 2, pp. 267-280, 1987.

[3] R. A. Jarrow and S. M. Turnbull, "Pricing derivatives on financial securities subject to credit risk," The Journal of Finance, vol. 50, no. 1, pp. 53-85, 1995.
[4] P. Klein, "Pricing Black-Scholes options with correlated credit risk," Journal of Banking \& Finance, vol. 20, no. 7, pp. 1211-1229, 1996.

[5] M.-W. Hung and Y.-H. Liu, "Pricing vulnerable options in incomplete markets," Journal of Futures Markets, vol. 25, no. 2, pp. 135-170, 2005.

[6] S.-L. Liao and H.-H. Huang, "Pricing Black-Scholes options with correlated interest rate risk and credit risk: an extension," Quantitative Finance, vol. 5, no. 5, pp. 443-457, 2005.

[7] P. Klein and M. Inglis, "Valuation of European options subject to financial distress and interest rate risk," Journal of Derivatives, vol. 6, no. 3, pp. 44-56, 1999.

[8] J.-H. Yoon and J.-H. Kim, "The pricing of vulnerable options with double Mellin transforms," Journal of Mathematical Analysis and Applications, vol. 422, no. 2, pp. 838-857, 2015.

[9] M.-K. Lee, S.-J. Yang, and J.-H. Kim, "A closed form solution for vulnerable options with Heston's stochastic volatility," Chaos, Solitons \& Fractals, vol. 86, pp. 23-27, 2016.

[10] G. Wang, X. Wang, and K. Zhou, "Pricing vulnerable options with stochastic volatility," Physica A: Statistical Mechanics and Its Applications, vol. 485, pp. 91-103, 2017.

[11] W. Xu, W. Xu, H. Li, and W. Xiao, "A jump-diffusion approach to modelling vulnerable option pricing," Finance Research Letters, vol. 9, no. 1, pp. 48-56, 2012.

[12] X. Wang, "Pricing vulnerable options with stochastic default barriers," Finance Research Letters, vol. 19, pp. 305-313, 2016.

[13] M. Han, X. Song, H. Niu, and S. Zhou, "Pricing vulnerable options with market prices of common jump risks under regime-switching models," Discrete Dynamics in Nature and Society, vol. 2018, Article ID 8545841, 15 pages, 2018.

[14] H. Niu, Y. Xing, and Y. Zhao, "Pricing vulnerable European options with dynamic correlation between market risk and credit risk," Journal of Management Science and Engineering, vol. 5, no. 2, pp. 125-145, 2020.

[15] C. Ma, Z. Ma, and S. Xiao, “A closed-form pricing formula for vulnerable European options under stochastic yield spreads and interest rates," Chaos, Solitons \& Fractals, vol. 123, pp. 59-68, 2019.

[16] M.-K. Lee and J.-H. Kim, "Pricing of defaultable options with multiscale generalized Heston's stochastic volatility," Mathematics and Computers in Simulation, vol. 144, pp. 235-246, 2018.

[17] C. Ma, S. Yue, and Y. Ren, "Pricing vulnerable European options under lévy process with stochastic volatility," Discrete Dynamics in Nature and Society, vol. 2018, Article ID 3402703, 16 pages, 2018.

[18] X. Han, "Valuation of vulnerable options under the double exponential jump model with stochastic volatility," Probability in the Engineering and Informational Sciences, vol. 33, no. 1, pp. 81-104, 2019.

[19] H. Niu and D. Wang, "Pricing vulnerable options with correlated jump-diffusion processes depending on various states of the economy," Quantitative Finance, vol. 16, no. 7, pp. 1129-1145, 2016.

[20] J. Yang, S. Zhou, H. Zhou, and K. Guo, "Pricing vulnerable option underJump-diffusion model with incomplete information," Discrete Dynamics in Nature and Society, vol. 2019, Article ID 5848375, 8 pages, 2019.

[21] P. Pasricha and A. Goel, "Pricing vulnerable power exchange options in an intensity based framework," Journal of Computational and Applied Mathematics, vol. 355, pp. 106-115, 2019. 
[22] B. B. Mandelbrot and J. W. Van Ness, "Fractional brownian motions, fractional noises and applications," SIAM Review, vol. 10, no. 4, pp. 422-437, 1968.

[23] A. W. Lo, "Long-term memory in stock market prices," Econometrica, vol. 59, no. 5, pp. 1279-1313, 1991.

[24] Z. Ding, C. W. J. Granger, and R. F. Engle, "A long memory property of stock market returns and a new model," Journal of Empirical Finance, vol. 1, no. 1, pp. 83-106, 1993.

[25] D. O. Cajueiro and B. M. Tabak, "Long-range dependence and multifractality in the term structure of LIBOR interest rates," Physica A: Statistical Mechanics and Its Applications, vol. 373, pp. 603-614, 2007.

[26] S. H. Kang and S.-M. Yoon, "Long memory properties in return and volatility: evidence from the Korean stock market," Physica A: Statistical Mechanics and Its Applications, vol. 385, no. 2, pp. 591-600, 2007.

[27] S. Lin, "Stochastic analysis of fractional Brownian motions," Stochastics andStochastics Reports, vol. 55, no. 1-2, pp. 121-140, 1995.

[28] L. C. G. Rogers, "Arbitrage with fractional Brownian motion," Mathematical Finance, vol. 7, no. 1, pp. 95-105, 1997.

[29] T. E. Duncan, Y. Hu, and B. Pasik-Duncan, "Stochastic calculus for fractional brownian motion I. Theory," SIAM Journal on Control and Optimization, vol. 38, no. 2, pp. 582-612, 2000.

[30] Y. Hu and B. Øksendal, "Fractional white noise calculus and applications to finance," Infinite Dimensional Analysis, Quantum Probability and Related Topics, vol. 6, no. 1, pp. 1-32, 2003.

[31] C. Necula, "Option pricing in a fractional Brownian motion environment," "Bucharest: The Academy of Economic Studies, vol. 27, no. 4, pp. 8079-8089, 2002.

[32] R. J. Elliott and J. van der Hoek, "A general fractional white noise theory and applications to finance," Mathematical Finance, vol. 13, no. 2, pp. 301-330, 2003.

[33] D. Nualart, The Malliavin Calculus and Related Topics, Springer BerlinHeidelberg, Berlin, Germany, 2006.

[34] W.-L. Xiao, W.-G. Zhang, X.-L. Zhang, and Y.-L. Wang, "Pricing currency options in a fractional Brownian motion with jumps," Economic Modelling, vol. 27, no. 5, pp. 935-942, 2010.

[35] C. Wang, S. Zhou, and J. Yang, "The pricing of vulnerable options in a fractional brownian motion environment," Discrete Dynamics in Nature and Society, vol. 2015, Article ID 579213, 10 pages, 2015.

[36] T. Björk and H. Hult, "A note on Wick products and the fractional Black-Scholesmodel," Finance and Stochastics, vol. 9, no. 2, pp. 197-209, 2005.

[37] C. Ei-Nouty, "The fractional mixed fractional Brownian motion," Statistics \& Probability Letters, vol. 65, no. 2, pp. 111-120, 2008.

[38] Y. Mishura, Stochastic Calculus for Fractional Brownian Motions and RelatedProcesses, Springer Press, Berlin, Germany, 2008.

[39] P. Cheridito, "Mixed fractional Brownian motion," Bernoulli, vol. 7, no. 6, pp. 913-934, 2001.

[40] W.-L. Xiao, W.-G. Zhang, X. Zhang, and X. Zhang, "Pricing model for equity warrants in a mixed fractional Brownian environment and its algorithm," Physica A: Statistical Mechanics and Its Applications, vol. 391, no. 24, pp. 6418-6431, 2012.

[41] F. Shokrollahi and A. Kılıçman, "Pricing currency option in a mixed fractional brownian motion with jumps environment," Mathematical Problems in Engineering, vol. 2014, Article ID 858210, 13 pages, 2014.
[42] W.-G. Zhang, Z. Li, Y.-J. Liu, and Y. Zhang, "Pricing European option under fuzzy mixed fractional brownian motion model with jumps," Computational Economics, vol. 58, no. 2, pp. 483-515, 2021.

[43] Z. Li and X. Wang, "Valuation of bid and ask prices for European options undermixed fractional Brownian motion," AIMS Mathematics, vol. 6, no. 7, pp. 7199-7214, 2021.

[44] W. Zhang, Z. Li, and Y. Liu, "Analytical pricing of geometric Asian power options onan underlying driven by a mixed fractional Brownian motion," Physica A: Statistical Mechanics and Its Applications, vol. 490, pp. 402-418, 2018.

[45] L. Sun, "Pricing currency options in the mixed fractional Brownian motion," Physica A: Statistical Mechanics and Its Applications, vol. 392, no. 16, pp. 3441-3458, 2013.

[46] F. Shokrollahi and A. Klllçman, "Actuarial approach in a mixed fractionalBrownian motion with jumps environment for pricing currency option," Advances in Difference Equations, vol. 1, pp. 257-264, 2015.

[47] R. Prakasa, "Pricing geometric Asian power options under mixed fractional Brownianmotion environment," Physica A: Statistical Mechanics and Its Applications, vol. 446, no. 1, pp. 92-99, 2016.

[48] M. Bladt and T. H. Rydberg, "An actuarial approach to option pricing under thephysical measure and without market assumptions," Insurance: Mathematics and Economics, vol. 22, no. 1 , pp. $65-73,1998$.

[49] H. Yan and S. Liu, "New method to option pricing for the general Black-Scholesmodel-an acturarial approach," Applied Mathematics and Mechanics, vol. 24, pp. 730-738, 2003.

[50] R. C. Merton, "Option pricing when underlying stock returns are discontinuous," Journal of Financial Economics, vol. 3, no. 1-2, pp. 125-144, 1976.

[51] W. Xu, W. Xu, and W. Xiao, "Pricing black-scholes options with correlated creditrisk and jump risk," Applied Economics Letters, vol. 22, no. 2, pp. 87-93, 2015. 